\title{
Video Article \\ A Label-free Technique for the Spatio-temporal Imaging of Single Cell Secretions
}

\author{
Deepa Raghu ${ }^{1}$, Joseph A. Christodoulides ${ }^{1}$, James B. Delehanty ${ }^{2}$, Jeff M. Byers ${ }^{1}$, Marc P. Raphael ${ }^{1}$ \\ ${ }^{1}$ Materials Science and Technology, Naval Research Laboratory \\ ${ }^{2}$ Center for Bio/Molecular Science and Engineering, Naval Research Laboratory
}

Correspondence to: Marc P. Raphael at marc.raphael@nrl.navy.mil

URL: https://www.jove.com/video/53120

DOI: doi: $10.3791 / 53120$

Keywords: Bioengineering, Issue 105, localized surface plasmon resonance (LSPR), nanoplasmonics, cell communication, secretion, signaling, hybridoma, single cell

Date Published: $11 / 23 / 2015$

Citation: Raghu, D., Christodoulides, J.A., Delehanty, J.B., Byers, J.M., Raphael, M.P. A Label-free Technique for the Spatio-temporal Imaging of Single Cell Secretions. J. Vis. Exp. (105), e53120, doi:10.3791/53120 (2015).

\section{Abstract}

Inter-cellular communication is an integral part of a complex system that helps in maintaining basic cellular activities. As a result, the malfunctioning of such signaling can lead to many disorders. To understand cell-to-cell signaling, it is essential to study the spatial and temporal nature of the secreted molecules from the cell without disturbing the local environment. Various assays have been developed to study protein secretion, however, these methods are typically based on fluorescent probes which disrupt the relevant signaling pathways. To overcome this limitation, a label-free technique is required.

In this paper, we describe the fabrication and application of a label-free localized surface plasmon resonance imaging (LSPRi) technology capable of detecting protein secretions from a single cell. The plasmonic nanostructures are lithographically patterned onto a standard glass coverslip and can be excited using visible light on commercially available light microscopes. Only a small fraction of the coverslip is covered by the nanostructures and hence this technique is well suited for combining common techniques such as fluorescence and bright-field imaging.

A multidisciplinary approach is used in this protocol which incorporates sensor nanofabrication and subsequent biofunctionalization, binding kinetics characterization of ligand and analyte, the integration of the chip and live cells, and the analysis of the measured signal. As a whole, this technology enables a general label-free approach towards mapping cellular secretions and correlating them with the responses of nearby cells.

\section{Video Link}

The video component of this article can be found at https://www.jove.com/video/53120/

\section{Introduction}

Inter-cellular communication is crucial for the regulation of many physiological activities both inside and outside the cell. A variety of proteins and vesicles can be secreted that subsequently trigger complex cellular processes such as differentiation, wound healing, immune response, migration, and proliferation. ${ }^{1-5}$ Malfunctions of inter-cellular signaling pathways have been implicated in numerous disorders including cancer, atherosclerosis, and diabetes, to name a few.

The optimal cell secretion assay should be capable of spatially and temporally mapping the secreted protein of interest without disrupting the relevant signaling pathways. In this way causal relationships between the concentration profiles and the response of the receiving cells can be inferred. Unfortunately, many of the most commonly used fluorescent-based techniques do not meet these criteria. Fluorescent fusion proteins can be used to tag the analyte within the cell but can disrupt the secretory pathway, or if secreted, results in a diffuse glow outside the cell which is difficult to quantify. Fluorescent immunosandwich-based assays are the most commonly used techniques for detecting cellular secretions but typically require the isolation of individual cells. ${ }^{6-11}$ In addition, the introduction of the sensing antibody typically halts or ends the experiment and the size of the antibody labels, $150 \mathrm{kDa}$ for $\operatorname{lgG}$, is an impediment to downstream signaling.

Because of these roadblocks it is preferable that a label-free technique be utilized to image protein secretions and amongst existing labelfree technologies, surface plasmon resonance (SPR) and localized surface plasmon resonance (LSPR) sensors are excellent candidates. ${ }^{12-17}$ These sensors have been widely used for analyte binding studies of proteins, exosomes and other biomarkers. ${ }^{18-24}$ In the case of LSPR, the plasmonic nanostructures can be patterned lithographically onto glass coverslips and excited using visible light via standard wide-field microscopy configurations. Due to their nanoscale footprint, the majority of the glass substrate is available for common imaging techniques such as bright-field and fluorescence microscopy making these probes well suited for integration with live-cell microscopy. ${ }^{25-28}$ We have demonstrated the real-time measurement of antibody secretions from hybridoma cells using functionalized gold plasmonic nanostructures with spatial and temporal resolutions of $225 \mathrm{msec}$ and $10 \mu \mathrm{m}$, respectively. The basic chip configuration is illustrated in Figure $1 .{ }^{28}$ The output light path of the 
microscope is split between a CCD camera used for imagery and a fiber-optically coupled spectrometer for the quantitative determination of fractional occupancy of a given array of nanostructures (Figure 2).

The protocol presented in this paper describes the experimental design for the real-time measurement of single cell secretions while simultaneously monitoring the response of the cells using the standard bright-field microscopy. The multidisciplinary approach includes the fabrication of nanostructures, functionalization of the nanostructures for the high affinity binding of analytes, surface optimization for both minimizing non-specific binding and characterizing kinetic rate constants using a commercial Surface Plasmon Resonance (SPR) instrument, integration of cell lines onto the substrate, and the analysis of imagery and spectral data. We anticipate this technique to be an enabling technology for the spatio-temporal mapping of cell secretions and their causal relationships with receiving cells.

\section{Nanostructure Fabrication}

1. Choose $25 \mathrm{~mm}$ diameter glass coverslips with an approximate thickness of $170 \mu \mathrm{m}$ (No. 1.5) as substrates for nanofabrication.

2. Immerse the coverslips in piranha solution (3:1 ratio of sulphuric acid and hydrogen peroxide) for at least $6 \mathrm{hr}$. Wash the piranha soaked coverslip with copious amount of ultrapure $18.2 \mathrm{M} \Omega$ deionized distilled water (DDW).

CAUTION: Piranha acid reacts violently with organic materials and must be handled with extreme care.

3. Deposit $10 \mathrm{~nm}$ chromium thin film on the coverslips by e-beam evaporation to avoid charge effects during the patterning and imaging of nanostructures.

4. Spin the first layer of bilayer resist consisting of ethyl lactate methyl methacrylate (MMA_EL6) copolymer at 2,000 rpm for 45 sec and then bake at $150^{\circ} \mathrm{C}$. Spin the second layer of poly-methyl methacrylate (950PMMA_A2) at 3,000 rpm for 45 sec then bake at $180{ }^{\circ} \mathrm{C}$.

5. Pattern the bilayer resist using the electron beam lithography (EBL) at $25 \mathrm{kV}$ with an area dose of $300 \mu \mathrm{C} / \mathrm{cm}^{2}$. Develop in isopropyl alcohol (IPA)/methyl isobutyl ketone (MIBK): 2/1 and rinse in IPA.

6. Deposit Ti $(5 \mathrm{~nm}) / \mathrm{Au}(80 \mathrm{~nm})$ film on the substrate using a e-beam evaporator.

7. Following the gold deposition, lift off the copolymer bilayer resist by soaking the substrate in acetone for $4 \mathrm{hr}$.

8. Inspect the substrate using the scanning electron microscope (SEM) to confirm nanostructure shape and size, remove the remaining chromium from the substrate via wet etch using CR-7 etchant for $60 \mathrm{sec}$ at RT and then rinse in DDW.

9. Design a center-to-center array spacing of $33 \mu \mathrm{m}$ to leave room for cell imaging between arrays. Pattern the nanostructures in $20 \times 20$ pattern for each array with a pitch of $300 \mathrm{~nm}$ using an e-beam writer. Each chip contains 300 arrays with a typical nanostructure dimension of $80 \pm 2.5$ $\mathrm{nm}$ height and $70 \pm 2.5 \mathrm{~nm}$ diameter.

10. Inspect a subset of arrays using the atomic force microscope (AFM) for the verification of size and uniformity.

11. Attach a support ring, typically silicon, to the back of the coverslip using a UV curing epoxy.

\section{Chip Cleaning and Application of Self-assembled Monolayer}

1. For cleaning and regenerating the chips, plasma ash at a power of $40 \mathrm{~W}$ in a $300 \mathrm{mTorr}$ mixture of $5 \%$ hydrogen, $95 \%$ argon for $45 \mathrm{sec}$ after cleaning the chamber for 5 min under the same conditions.

2. Functionalize the gold nanostructures immediately after plasma ashing by immersing the chip in a two-component ethanolic thiol solution consisting of a 3:1 ratio of $\mathrm{SH}-\left(\mathrm{CH}_{2}\right)_{8}-\mathrm{EG}_{3}-\mathrm{OH}(\mathrm{SPO})$ and a component with either an amine or carboxyl functional group, namely, $\mathrm{SH}-$ $\left(\mathrm{CH}_{2}\right)_{11}-\mathrm{EG}_{3}-\mathrm{NH}_{2}(\mathrm{SPN})$ or $\mathrm{SH}-\left(\mathrm{CH}_{2}\right)_{11}-\mathrm{EG}_{3}-\mathrm{COOH}(\mathrm{SPC})$.

3. Leave the chip in the thiol solution $\mathrm{O} / \mathrm{N}$ to form a self-assembled monolayer (SAM).

4. Rinse the chip with ethanol and dry with nitrogen gas.

5. If needed, store the functionalized chip for up to 2 weeks at $4{ }^{\circ} \mathrm{C}$

6. When ready for use react the SPN or SPC component with the ligand using a chemistry depending on the ligand of choice (see below) Note: The chips can be regenerated and re-functionalized dozens of times. A given chip can be used for periods that range from 6 months to over a year. The spectra measured on a given array are reliably reproduced after repeated regenerations by plasma ashing, followed by biofunctionalization. ${ }^{29}$

\section{Surface Functionalization and Kinetic Characterization}

Note: Use the functionalized chip in the commercial SPR instrument to characterize the kinetic rate constants between the ligand and the analyte, as well as to study the resistance of SAM to non-specific binding. There is a wide range of flow rates and microfluidic designs that allow for efficient surface functionalization. Since we have a commercially available SPR we standardized around its recommended flow rates. We note that these flow rates are typical of all SPR instruments and so are not restrictive. The SPR instrument is not a necessity since all functionalization can be done directly on the LSPR chip, but it did reduce our work load because it is a multiplexed instrument whereas our LSPR microfluidic setup is not.

1. Functionalize a commercial bare gold chip with the SAM as described in Section 2.

2. If using an SPC based SAM, activate the carboxyl group with a 1:1 mixture of $133 \mathrm{mM}$ of 1-ethyl-3-(3-dimethylaminopropyl) carbodiimide (EDC) and $33 \mathrm{mM}$ of $\mathrm{N}$-hydroxysuccinimide (NHS) in DDW for $10 \mathrm{~min}$.

3. Conjugate the activated carboxyl group with the antibody/ligand of interest for $300 \mathrm{sec}$ using a flow rate of $30 \mu \mathrm{l} / \mathrm{min}$. Prepare the ligands in $\mathrm{pH} 6$ phosphate buffer, typically, but this can vary depending on the molecule.

4. After the ligand conjugation, flow $0.1 \mathrm{M}$ ethanolamine in phosphate-buffered saline (PBS) as a deactivator step for 300 sec at a rate of $30 \mu \mathrm{l} /$ min. Ethanolamine helps in minimizing the non-specific binding.

5. Introduce the analyte of interest at a flow rate of $100 \mu \mathrm{l} / \mathrm{min}$ using a range of concentrations and calculate the kinetic rate constants using a kinetic analysis software. 
6. If non-specific binding is problematic, increase the ratio of SPO to SPC or SPN.

\section{LSPR General Settings}

1. Microscope settings:

1. Use a $100 \mathrm{~W}$ Halogen lamp to Koehler illuminate the sample. Use a long pass filter (typically $593 \mathrm{~nm}$ cutoff) in the light path to eliminate wavelengths that do not contribute to the resonance shift (Figure 2).

2. For the LSPRi data collection, use an inverted microscope with a $40 \mathrm{X}$ oil immersion objective (1.4 NA) and a thermoelectrically cooled 16 bit CCD camera.

3. Place a beam splitter at the output port of the microscope to obtain Imagery and spectra simultaneously.

4. Set the temperature controlled microscope stage to $37^{\circ} \mathrm{C}$ and equilibrate for $4 \mathrm{hr}$.

5. Incorporate an additional incubation assembly on the microscope to regulate the $\mathrm{CO}_{2}$ concentration and humidity at $5 \%$ and $95 \%$, respectively.

2. Chip preparation and mounting:

1. Functionalize the LSPR chip as described in Section 2 with the optimal two-component SAM ratios determined from the SPR experiments.

2. Load the chip within a custom made microfluidic holder as follows. Place the chip on an aluminum bottom piece. Sandwich the chip between this bottom piece and a silicone gasket and a clear plastic top piece. Use 4 screws to clamp the assembly.

3. For a typical SPC-based thiol application, drop coat $300 \mu \mathrm{l}$ a 1:1 mixture of $133 \mathrm{mM}$ of EDC and $33 \mathrm{mM}$ of NHS in DDW to activate the carboxyl groups of the SPC thiol component.

4. Wait for $10 \mathrm{~min}$ and manually rinse the surface with $10 \mathrm{mM}$ PBS.

5. Conjugate the activated carboxyl group with the ligand (typically an antibody or antibody fragment) of interest by drop coating $300 \mu \mathrm{l}$ of ligand solution.

6. Wait for $30 \mathrm{~min}$ and manually rinse with $10 \mathrm{mM}$ PBS.

7. Drop coat $300 \mu \mathrm{l}$ of $0.1 \mathrm{M}$ ethanolamine in PBS on the chip to minimize nonspecific binding. Wait for $10 \mathrm{~min}$.

8. Wash the ethanolamine with PBS containing $0.005 \%$ Tween 20 (PBS-T20).

9. Place a quartz piece above the chip to reduce fluctuations in the data related to a changing meniscus.

10. Keep the chip wet with PBS-T20 buffer while mounting on microscope.

11. Place the LSPR chip assembly firmly into the heated stage sample holder and attach microfluidic tubing.

12. Attach the microfluidics tubing to the assembly and flow buffer (or serum free media for cell studies) until a steady state is reached.

13. Allow the assembly and microscope to equilibrate for at least $2 \mathrm{hr}$.

14. Align the chip using joystick so that the central array is aligned with the fiber optic for spectroscopy. Spectroscopic data is taken using a spectrometer and spectral analysis software.

15. Keep arrays in focus throughout the experiment by using software autofocus, Zeiss Definite Focus or equivalent autofocus device.

\section{LSPR Imaging of Anti-c-myc Secretions from 9E10 Hybridoma Cells}

Note: The hybridoma cell line used for this study express anti-c-myc antibody constitutively and hence do not require a chemical trigger

1. Functionalize the nanostructures with c-myc peptide. This has a $\mathrm{K}_{\mathrm{D}}$ value of $1.77 \mathrm{nM}$ for the anti-c-myc antibodies secreted by clone $9 \mathrm{E} 10$ hybridoma cells.

2. Culture the hybridoma cells in complete growth medium with $10 \%$ fetal bovine serum (FBS) and $1 \%$ antibiotic in a $\mathrm{T} 75$ flask at $37{ }^{\circ} \mathrm{C}$ under $5 \% \mathrm{CO}_{2}$. Maintain a cell density of $4 \times 10^{5} \mathrm{cells} / \mathrm{ml}$.

3. For the cell secretion studies, pellet cells from the T75 flask by centrifugation, wash twice with RPMI-1640 serum free media (SFM) to remove the secreted antibodies and adjust the cell density to $4 \times 10^{6} \mathrm{cells} / \mathrm{ml}$.

4. Harvest the cells and test for viability before introducing them on to the LSPR chips.

5. Introduce $50 \mu \mathrm{l}$ of the cell solution manually onto the LSPR chips with a micropipette. After few minutes 25 to 50 cells adhere to the surface of the LSPR chips.

6. Wash away the remaining cells in solution with fresh SFM using the microfluidic perfusion system

7. Select the LSPR arrays for imaging which are close, within $10 \mu \mathrm{m}$, but not overlapping with the cells.

8. To ensure that the signal is specific to the secreted anti-c-myc antibodies introduce the cell culture media with and without the antibodies present as well as with the antibodies but with their binding sites blocked by the presence of a saturating concentration of c-myc peptide in the solution.

9. Calibrate the sensors at the end of each run with a saturating solution of anti-c-myc antibodies ( $250 \mathrm{nM}$ ). This helps in normalizing the response of the sensors and determine fractional occupancy based on the biofunctionalization profile of each run.

10. Correct the drift in the $X$ and $Y$ direction using image alignment software.

\section{Representative Results}

In a typical live cell secretion study there are multiple modes of data collection taking place. Figure 3 shows an overlay of an LSPRi image, which highlights the square arrays, and a transmitted light illuminated image which highlights the cell at lower left. Data is typically collected over a 3-hr period followed by the introduction of a saturating solution of the analyte for the normalization calculation described below. Fluorescence imagery can also be integrated into the data collection routine by the automated switching of a filter cube. In Figure $\mathbf{4}$ a cell stained with the fluorescent membrane dye rhodamine DHPE exhibits lamellipodia-like extensions (arrows). If such extensions were to overlap with the arrays they would give a false-positive for protein secretion. Having multiple modes of imagery can help identify such occurrences. 
Figure 5 shows spectrometry data before and after the introduction of a saturating solution ( $400 \mathrm{nM})$ of commercially purchased anti-c-myc antibodies to the c-myc functionalized arrays. No cells were present in this experiment. The spectrum displays both a red shift and an increase in intensity. The difference between the areas under the two curves results in an increase in the array image intensity in LSPRi mode on the CCD camera. A non-linear least squares data analysis approach has been developed to infer fractional occupancy of surface bound ligands from the spectra. $^{30,31}$

At the end of the experiment, the saturated intensity values (i.e., fractional occupancy $\approx 1$ ) are used to calculate a normalized response for each array using the following formula:

Where are the normalized intensity at time point $t$, initial intensity at the start of the experiment, final saturated intensity, and measured intensity of the array at time point $t$, respectively.

Normalized values from two arrays are shown in Figure 6. One array was within $10 \mu \mathrm{m}$ of the cell under investigation while the other, used as a control, was a distance of $130 \mu \mathrm{m}$ from the cell. The sudden increase in the normalized response of the array closest to the cell relative to the flat response of the control array is indicative of a localized burst of secreted antibodies.

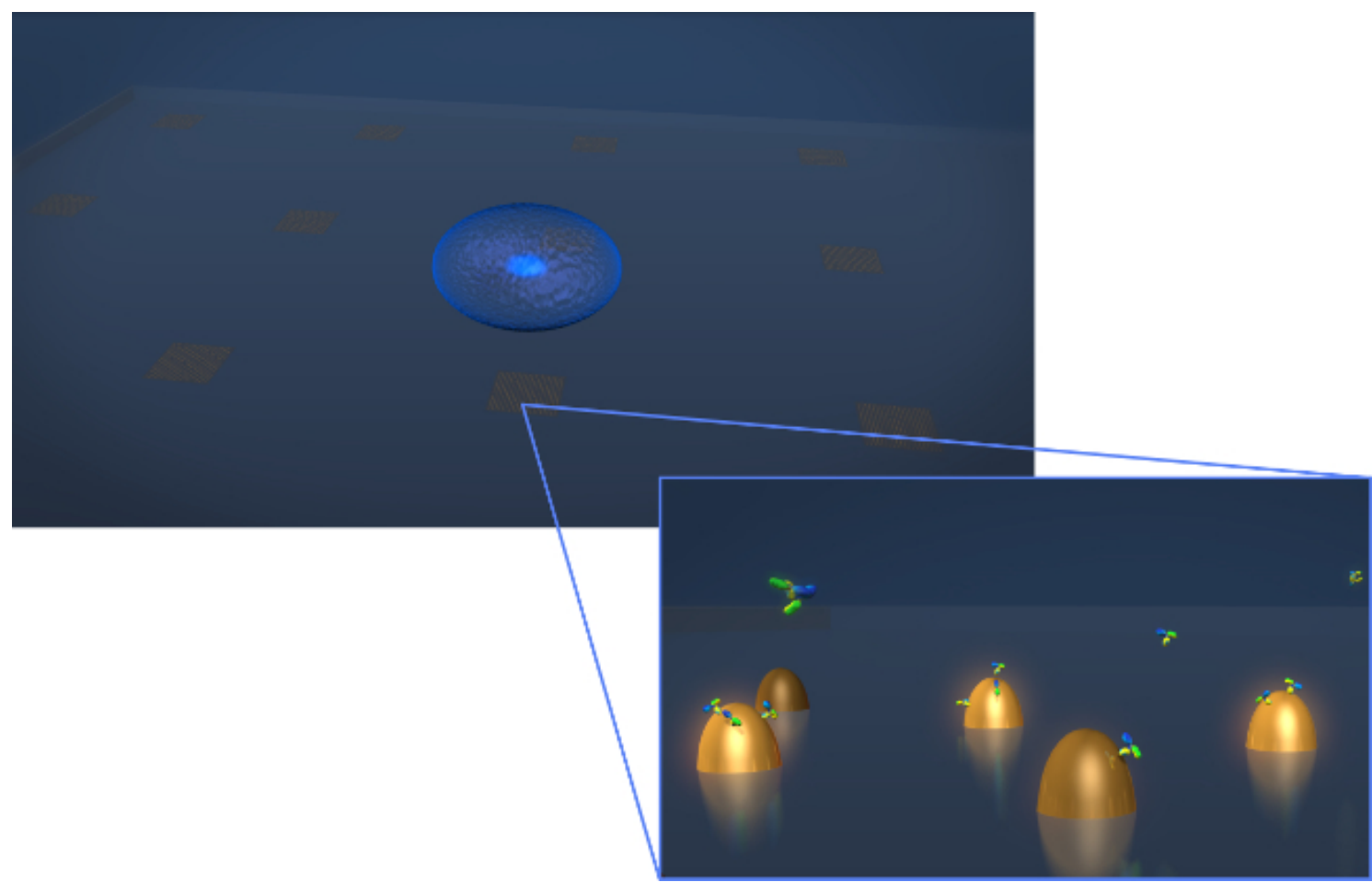

Figure 1. Sensor Design. A drawing depicting the geometry of a typical live cell secretion experiment. The cell (blue spheroid) is deposited on to the LSPR chip which contains arrays of biofunctionalized gold nanostructures. In the zoomed-in view, the cell secretion of interest, in this case antibodies shown as Y-shaped molecules, is measured as they bind to the surface of the functionalized nanostructures. Please click here to view a larger version of this figure. 


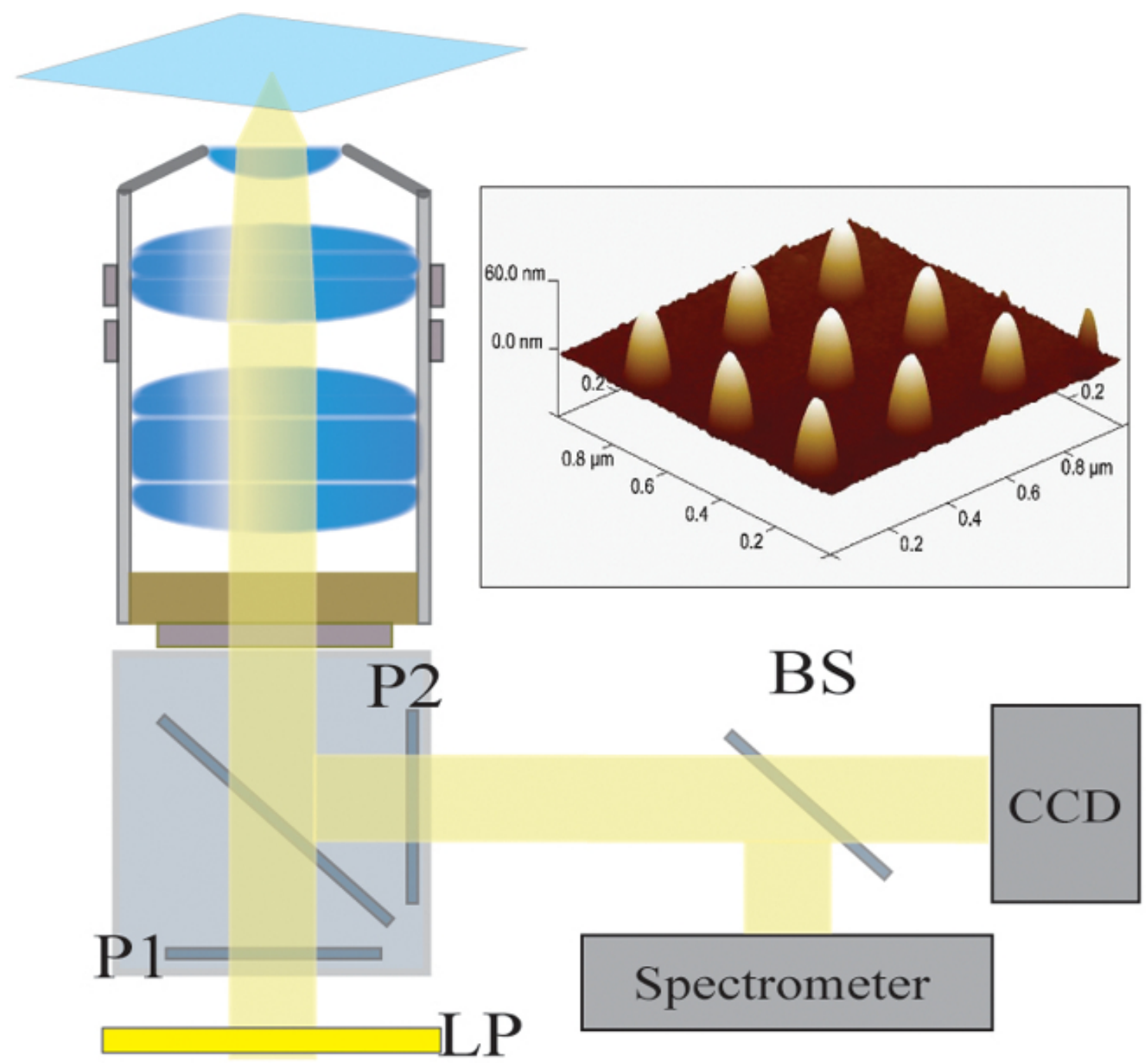

Figure 2. Optical Setup. The illuminated light from a halogen lamp is first filtered by a long pass filter (LP). The light is linearly polarized (P1) and illuminates the sample via a 40X/1.4 NA objective. The scattered light is collected by the objective and passed through a crossed polarizer (P2). A 50/50 beam splitter (BS) is inserted into the collected light path for simultaneous spectroscopic and imagery analysis. Top Right: An atomic force microscopy image of 9 individual nanostructures separated by a pitch of $300 \mathrm{~nm}$. Please click here to view a larger version of this figure. 


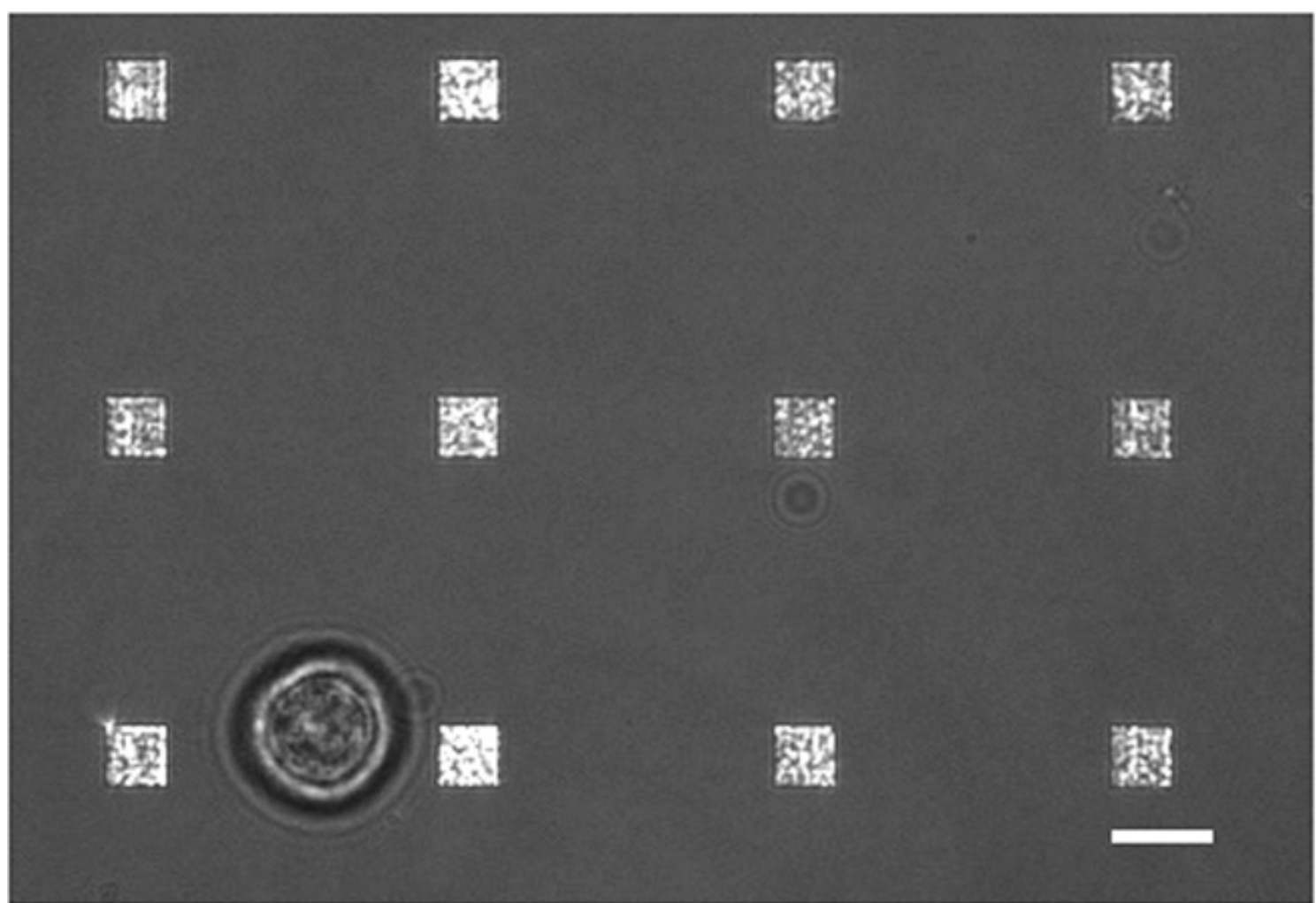

Figure 3. Live Cell LSPRi Study. A merged transmitted light and LSPRi image showing a single hybridoma cell (lower left) surrounded by 12 arrays. This is a contrast enhanced image. Scale bar is $10 \mu \mathrm{m}$. Please click here to view a larger version of this figure. 


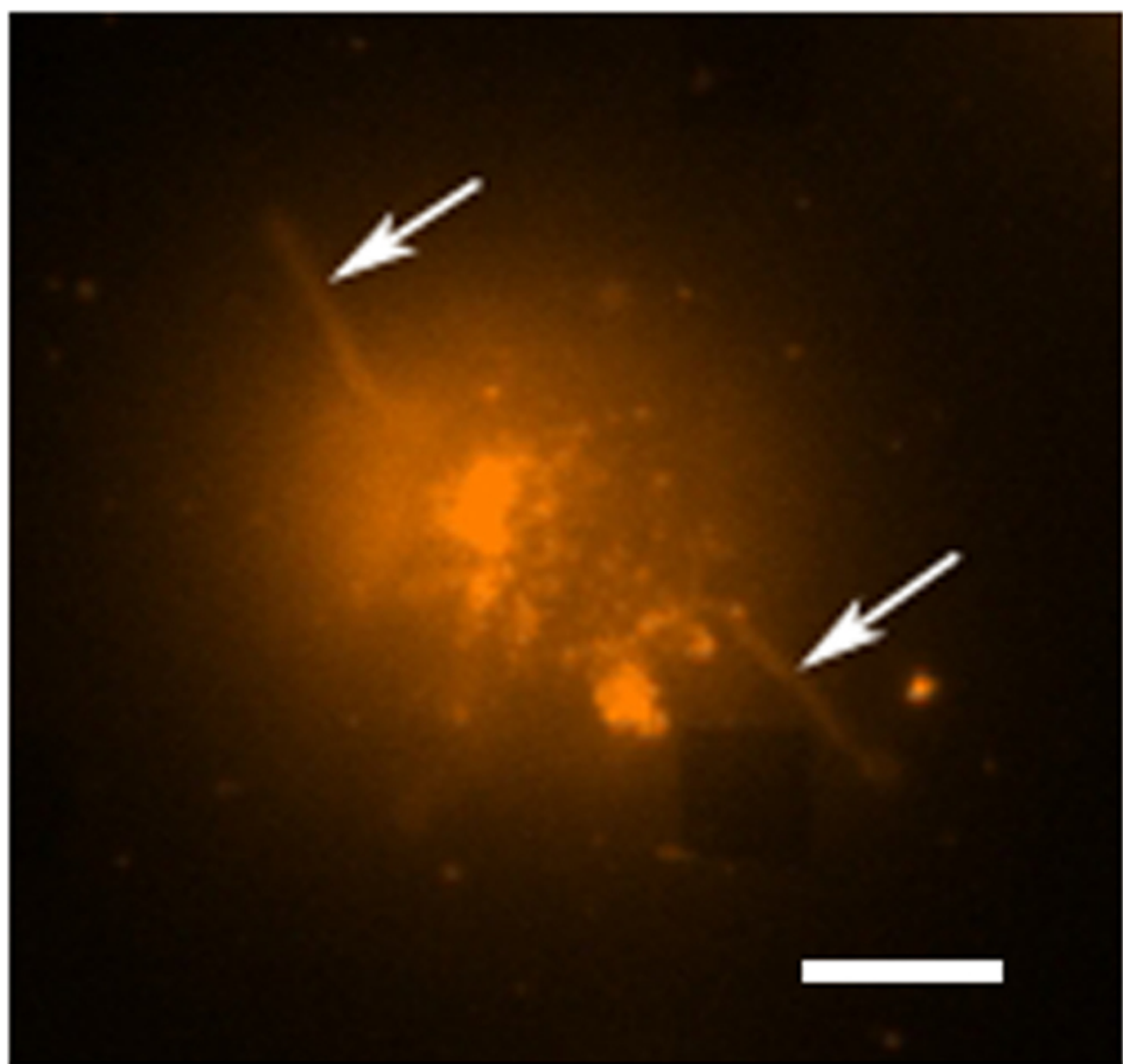

Figure 4. Live Cell Fluorescence Study. A fluorescent false color image of a single hybridoma cell stained with rhodamine DHPE, which is a membrane dye. In fluorescent imaging mode the arrays are not generally visible, however, a nearby array is observable here as a black square in the lower right corner. The cell can be seen to be separated from the array although tentacle-like extensions (possibly filopodia or lamellipodia) are extending outward from the cells (arrows). Scale bar is $10 \mu \mathrm{m}$. Please click here to view a larger version of this figure. 


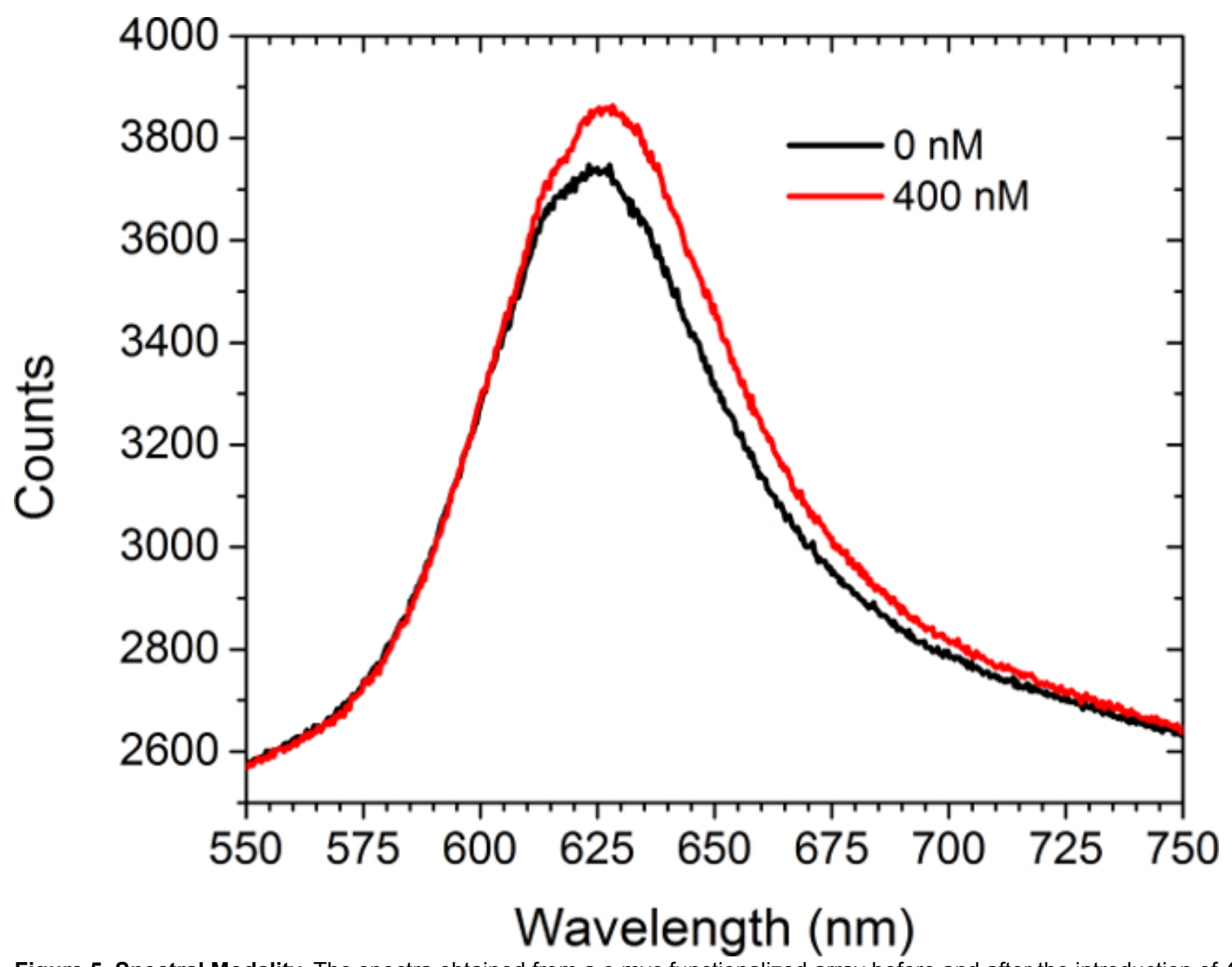

Figure 5. Spectral Modality. The spectra obtained from a c-myc functionalized array before and after the introduction of $400 \mathrm{nM}$ solution of antic-myc antibodies. No cells were present in this study. Please click here to view a larger version of this figure. 


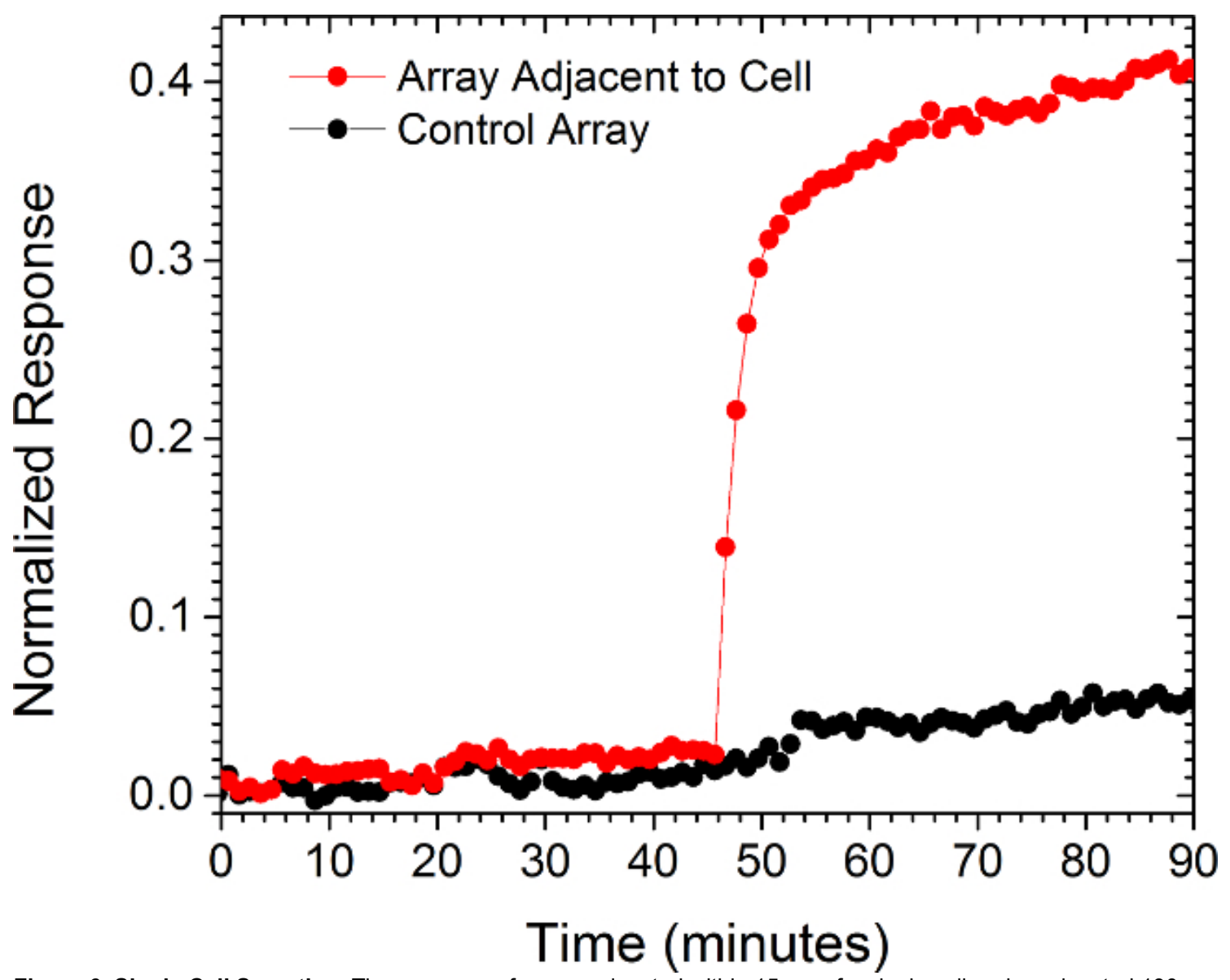

Figure 6. Single Cell Secretion. The response of an array located within $15 \mu \mathrm{m}$ of a single cell and one located $130 \mu \mathrm{m}$ away (Control). Scale bar is $10 \mu \mathrm{m}$. Please click here to view a larger version of this figure.

\section{Discussion}

The LSPR imaging technique described in this work has numerous advantages over more traditional methodologies for detecting cell secretions. First, the time resolution of our technique is on the order of seconds whereas the commercial alternative, an immunosandwhich assay known as EliSpot, has a typical time resolution of 2 to 3 days. ${ }^{7,32}$ As a result we were able to resolve sudden changes in the rate of protein secretion, such as that shown in Figure 6. Second, having arrays distributed over the chip allows for the secreted signal to be tracked in space and time which enables more rigorous comparisons to diffusion-based models of cell secretion. In addition, arrays like the control array shown in Figure 6 can be used to subtract out global changes in the image that typically arise from instrumental factors such as focus drift. Third, our technique requires no modification of the cells. If desired, the experiment can incorporate commonly used tags such as fluorescent proteins, but if there is concern that such tags may negatively affect cell viability or homeostasis the label-free nature of our approach does not require them. Fourth, using the spectroscopic data we have demonstrated that quantitative information regarding the fractional occupancy of surface bound ligands can be calculated.

There are numerous alternative methods to EBL for fabricating metallic nanoparticles. However, we have found that the EBL provides considerable flexibility for optimizing nanostructure and array dimensions to best suit the optics and the cells under investigation. Also critical is the fact that the chips can be readily regenerated by plasma ashing. In this way, a typical chip can be used dozens of times. Biofunctionalization details must be modified for the specific application. The protocol presented here conjugated the surface with relatively small c-myc peptide ligands. Larger ligands such as whole antibodies typically require more spacing and thus a higher SPO to SPN/SPC ratio. Regardless, a well formed SAM layer is essential for preventing non-specific binding in live-cell experiments. In general, larger molecular weight analytes are more readily detected by LSPR. Thus, in its single-cell manifestation, this technique may not be appropriate for detecting the secretion of small proteins, such as cytokines.

The current setup has been used for studying individual non-adherent cells. There are significant number of secreted signaling proteins and vesicles to which the results reported in this work are directly applicable. For example carcinoembryonic antigen (CEA) which for decades now has been a diagnostic marker for cancer. Colon cancer cells are known to secrete CEA at the rates of thousands of molecules/cell/hr and the molecular weight is $180 \mathrm{kDa}$ which exceeds that of $\mathrm{IgG}$ antibodies. CEA is believed to be involved in autocrine and paracrine signaling pathways but the spatio-temporal nature of these secretions have never been measured. Our technique can directly address these signaling questions. An extension of this work will be to measure the spatio-temporal nature of CEA secretion from single cells. ${ }^{33}$ Future work will also focus on integrating LSPRi with two and three dimensional cell cultures of adherent cells. By incorporating multiplexed arrays capable of detecting a 
number of secreted proteins in parallel, this technique has the potential to open a new window into cell secretions and how they influence neighboring cells.

\section{Disclosures}

We thank George Anderson for helpful comments and discussions. This work was supported by the Naval Research Laboratory's Institute for Nanoscience and the National Research Council Research Associateship Award.

\section{Acknowledgements}

The authors have nothing to disclose.

\section{References}

1. Ludwig, A.-K., \& Giebel, B. Exosomes: Small vesicles participating in intercellular communication. The International Journal of Biochemistry \& Cell Biology. 44, 11-15, doi:10.1016/j.biocel.2011.10.005 (2012).

2. Friedl, P., \& Gilmour, D. Collective cell migration in morphogenesis, regeneration and cancer. Nature Reviews Molecular Cell Biology. 10 445-457, doi:10.1038/nrm2720 (2009).

3. Letterio, J. J., \& Roberts, A. B. Regulation of immune responses by TGF-beta. Annual Review of Immunology. 16, 137-161, doi:10.1146/ annurev.immunol.16.1.137 (1998).

4. Werner, S., \& Grose, R. Regulation of wound healing by growth factors and cytokines. Physiological Reviews. 83, 835-870, doi:10.1152/ physrev.00031.2002 (2003).

5. Werner, S., Krieg, T., \& Smola, H. Keratinocyte-fibroblast interactions in wound healing. Journal of Investigative Dermatology. 127, 998-1008, doi:10.1038/sj.jid.5700786 (2007).

6. Bailey, R. C., Kwong, G. A., Radu, C. G., Witte, O. N., \& Heath, J. R. DNA-encoded antibody libraries: A unified platform for multiplexed cell sorting and detection of genes and proteins. Journal of the American Chemical Society. 129, 1959-1967, doi:10.1021/ja065930i (2007).

7. Gazagne, A. et al. A Fluorospot assay to detect single T lymphocytes simultaneously producing multiple cytokines. Journal of Immunological Methods. 283, 91-98, doi:10.1016/j.jim.2003.08.013 (2003).

8. Han, Q. et al. Polyfunctional responses by human T cells result from sequential release of cytokines. Proceedings of the National Academy of Sciences of the United States of America. 109, 1607-1612, doi:10.1073/pnas.1117194109 (2012).

9. Han, Q., Bradshaw, E. M., Nilsson, B., Hafler, D. A., \& Love, J. C. Multidimensional analysis of the frequencies and rates of cytokine secretion from single cells by quantitative microengraving. Lab on a Chip. 10, 1391-1400, doi:10.1039/b926849a (2010).

10. Ma, C. et al. A clinical microchip for evaluation of single immune cells reveals high functional heterogeneity in phenotypically similar $\mathrm{T}$ cells. Nature Medicine. 17, 738-743, doi:10.1038/nm.2375 (2011).

11. Shirasaki, Y. et al. Real-time single-cell imaging of protein secretion. Scientific Reports. 4, 8, doi:10.1038/srep04736 (2014).

12. Milgram, S. et al. On chip real time monitoring of B-cells hybridoma secretion of immunoglobulin. Biosensors and Bioelectronics. 26, 2728-2732, doi:10.1016/j.bios.2010.09.044 (2011).

13. Abbas, A., Linman, M. J., \& Cheng, Q. A. New trends in instrumental design for surface plasmon resonance-based biosensors. Biosensors \& Bioelectronics. 26, 1815-1824, doi:10.1016/j.bios.2010.09.030 (2011).

14. Ermakova, A. et al. Detection of a Few Metallo-Protein Molecules Using Color Centers in Nanodiamonds. Nano Letters. 13, 3305-3309, doi:10.1021/nl4015233 (2013).

15. Haes, A. J., \& Van Duyne, R. P. A nanoscale optical blosensor: Sensitivity and selectivity of an approach based on the localized surface plasmon resonance spectroscopy of triangular silver nanoparticles. Journal of the American Chemical Society. 124, 10596-10604, doi:10.1021/ja020393x (2002).

16. Horowitz, V. R., Aleman, B. J., Christle, D. J., Cleland, A. N., \& Awschalom, D. D. Electron spin resonance of nitrogen-vacancy centers in optically trapped nanodiamonds. Proceedings of the National Academy of Sciences of the United States of America. 109, 13493-13497, doi:10.1073/pnas.1211311109 (2012).

17. Sepulveda, B., Angelome, P. C., Lechuga, L. M., \& Liz-Marzan, L. M. LSPR-based nanobiosensors. Nano Today. 4, 244-251, doi:10.1016/ j.nantod.2009.04.001 (2009).

18. Barbillon, G. et al. Biological and chemical gold nanosensors based on localized surface plasmon resonance. Gold Bulletin. 40, 240-244, doi:10.1007/BF03215587 (2007).

19. Endo, T. et al. Multiple label-free detection of antigen-antibody reaction using localized surface plasmon resonance-based core-shell structured nanoparticle layer nanochip. Analytical Chemistry. 78, 6465-6475, doi:10.1021/ac0608321 (2006).

20. Endo, T., Kerman, K., Nagatani, N., Takamura, Y., \& Tamiya, E. Label-free detection of peptide nucleic acid-DNA hybridization using localized surface plasmon resonance based optical biosensor. Analytical Chemistry. 77, 6976-6984, doi:10.1021/ac0513459 (2005).

21. Haes, A. J., Hall, W. P., Chang, L., Klein, W. L., \& Van Duyne, R. P. A localized surface plasmon resonance biosensor: First steps toward an assay for Alzheimer's disease. Nano Letters. 4, 1029-1034, doi:10.1021/nl049670j (2004).

22. Jonsson, M. P., Jonsson, P., Dahlin, A. B., \& Hook, F. Supported lipid bilayer formation and lipid-membrane-mediated biorecognition reactions studied with a new nanoplasmonic sensor template. Nano Letters. 7, 3462-3468, doi:10.1021/nl072006t (2007).

23. Park, J. H. et al. A regeneratable, label-free, localized surface plasmon resonance (LSPR) aptasensor for the detection of ochratoxin A. Biosensors, \& Bioelectronics. 59, 321-327, doi:10.1016/j.bios.2014.03.059 (2014).

24. Mayer, K. M., Hao, F., Lee, S., Nordlander, P., \& Hafner, J. H. A single molecule immunoassay by localized surface plasmon resonance. Nanotechnology. 21, doi:10.1088/0957-4484/21/25/255503 (2010).

25. Endo, T., Yamamura, S., Kerman, K., \& Tamiya, E. Label-free cell-based assay using localized surface plasmon resonance biosensor. Analytica Chimica Acta. 614, 182-189, doi:10.1016/j.aca.2008.03.022 (2008).

26. Huang, Y. X., Cai, D., \& Chen, P. Micro- and Nanotechnologies for Study of Cell Secretion. Analytical Chemistry. 83, 4393-4406, doi:10.1021/ ac200358b (2011). 
27. Oh, B. R. et al. Integrated Nanoplasmonic Sensing for Cellular Functional Immunoanalysis Using Human Blood. ACS Nano. 8, 2667-2676, doi:10.1021/nn406370u (2014).

28. Raphael, M. P., Christodoulides, J. A., Delehanty, J. B., Long, J. P., \& Byers, J. M. Quantitative Imaging of Protein Secretions from Single Cells in Real Time. Biophysical Journal. 105, 602-608, doi:10.1016/j.bpj.2013.06.022 (2013).

29. Raphael, M. P. et al. A New Methodology for Quantitative LSPR Biosensing and Imaging. Analytical Chemistry. 84, 1367-1373, doi:10.1021/ ac2023266 (2011).

30. Raphael, M. P. et al. Quantitative LSPR imaging for biosensing with single nanostructure resolution. Biophysical Journal. 104, 30-36, doi:10.1016/j.bpj.2012.11.3821 (2013).

31. Raphael, M. P. et al. A new methodology for quantitative LSPR biosensing and imaging. Analytical Chemistry. 84, 1367-1373, doi:10.1021/ ac2023266 (2012).

32. Henn, A. D. et al. Modulation of single-cell IgG secretion frequency and rates in human memory B cells by CpG DNA, CD40L, IL-21, and cell division. Journal of Immunology. 183, 3177-3187, doi:10.4049/jimmunol.0804233 (2009).

33. Bramswig, K. H. et al. Soluble Carcinoembryonic Antigen Activates Endothelial Cells and Tumor Angiogenesis. Cancer Research. 73, 6584-6596, doi:10.1158/0008-5472.can-13-0123 (2013). 